\title{
Stratégies et styles rédactionnels de l'article de recherche : les ressources de l'utilisateur non-natif devant publier en anglais
}

\section{Claude Sionis}

\section{OpenEdition}

\section{Journals}

Édition électronique

URL : http://journals.openedition.org/asp/3035

DOI : $10.4000 /$ asp.3035

ISSN : 2108-6354

Éditeur

Groupe d'étude et de recherche en anglais de spécialité

\section{Édition imprimée}

Date de publication : 1 décembre 1997

Pagination : 207-222

ISSN : 1246-8185

\section{Référence électronique}

Claude Sionis, «Stratégies et styles rédactionnels de l'article de recherche : les ressources de I'utilisateur non-natif devant publier en anglais », ASp [En ligne], 15-18| 1997, mis en ligne le 31 juillet 2012, consulté le 30 avril 2019. URL : http://journals.openedition.org/asp/3035; DOI : 10.4000/ asp.3035

Ce document a été généré automatiquement le 30 avril 2019.

Tous droits réservés 


\title{
Stratégies et styles rédactionnels de l'article de recherche : les ressources de l'utilisateur non-natif devant publier en anglais
}

\author{
Claude Sionis
}

1 La communication et sa présente transcription sont une adaptation autorisée de l'article du même auteur, "Communication strategies in the writing of scientific research articles by non-native users of English ", paru dans la revue English For Specific Purposes 14/ 2. Copyright ( 1995 The American University.

\section{Introduction}

2 L'étude porte sur le comportement rédactionnel de deux groupes témoins de chercheurs de langue maternelle français: collègues enseignants-chercheurs non-linguistes de l'École Centrale de Nantes. Les deux groupes qui se sont adressés au professeur d'ESP/EST à la suite d'un refus de publication d'article sont décrits dans le tableau 0 .

Tableau 0. Groupes de chercheurs

\begin{tabular}{|l|l|l|}
\hline & GROUPE 1 (2 chercheurs) & GROUPE 2 ( 3 chercheurs) \\
\hline Âge & 55 et 56 ans & $30,31,32$ ans \\
\hline Domaine & calcul des structures & géotechnique \\
\hline
\end{tabular}




\begin{tabular}{|c|c|c|}
\hline $\begin{array}{l}\text { Titre } \\
\text { l'article }\end{array}$ & $\begin{array}{l}\text { "Dynamic condensation : free } \\
\text { modes method" } \\
7128 \text { mots }\end{array}$ & $\begin{array}{l}\text { «Resolution of elastoplastic constitutive } \\
\text { relations : application to fibre-reinforced } \\
\text { sand» } \\
5954 \text { mots }\end{array}$ \\
\hline Caractéristiques & $\begin{array}{l}\text { - Réputation de chercheurs bien } \\
\text { assise en France. } \\
\text { - Formation traditionnelle en } \\
\text { anglais, du type "structuro- } \\
\text { lexicale » (années 60). } \\
\text { - Connaissance "référentielle» } \\
\text { de l'anglais. } \\
\text { - Incapables d'échanger de } \\
\text { manière interactive avec leurs } \\
\text { pairs anglophones. } \\
\text { - Ont déjà publié en anglais mais } \\
\text { avec l'aide considérable (et } \\
\text { onéreuse) de traducteurs. } \\
\text { - Souhaitent bénéficier de } \\
\text { recettes, de «trucs»: attitude " } \\
\text { use and forget », ce que R. Gardner } \\
\text { et W. Lambert (1959) appellent } \\
\text { «motivation instrumentale ». }\end{array}$ & $\begin{array}{l}\text { - Ont déjà publié en français, cet article } \\
\text { est leur première publication (et co- } \\
\text { rédigée) en anglais. } \\
\text { - Ont bénéficié d'un enseignement } \\
\text { d'anglais du type communicationnel ( } \\
\text { Communicative Language Teaching, CLT) : } \\
\text { approche interactive, programme } \\
\text { "sémantique ", recherche de la fluidité } \\
\text { de l'expression, etc. } \\
\text { - Fortement motivés envers l'anglais; } \\
\text { obligation de publier en anglais acceptée } \\
\text { dès le début de leur carrière de } \\
\text { chercheurs; éventualité de rejoindre une } \\
\text { équipe anglophone. } \\
\text { - Illustrent ce que R. Gardner et W. } \\
\text { Lambert (1959) appellent "motivation } \\
\text { intégrative ". }\end{array}$ \\
\hline
\end{tabular}

3 Ces rédacteurs en tant que groupes présentent des points communs et des différences.

\section{Points communs :}

- Les 2 groupes sont francophones de langue, de culture et de formation (sciences de la matière).

- Ils veulent publier dans des revues anglophones qui font autorité dans leurs domaines d'application.

- Ils rédigent en équipe.

- Ils n'ont pas suivi, en formation initiale, de cours d'ESP/EST centrés ou non sur leur domaine d'application ultérieur. La formation en anglais a été du type « généraliste » (langue non spécialisée).

4 La cause du refus de publication est, dans les deux cas, et au premier abord, l'aspect formel des articles: essentiellement la langue écrite utilisée jugée inadéquate par les évaluateurs natifs ou quasiment natifs.

5 Le professeur d'EST s'est donc trouvé dans la position d'un médecin légiste qui pratiquerait une autopsie et la commenterait en présence des meurtriers.

\section{Différences :}

6 Comme on l'a vu dans les fiches descriptives (tableau 0), celles-ci sont nombreuses mais l'étude s'est surtout attachée au type d'enseignement d'anglais reçu par les deux groupes, à cet «héritage pédagogique » et à l'attitude qui a été créée vis-à-vis de la L2 et de son utilisation en contexte professionnel.

7 Il faut préciser d'emblée que les rédacteurs ont dû, dans un premier temps, faire face seuls aux difficultés de leur tâche. Les formulations proposées sont souvent les seules 
qu'ils connaissaient. Ils se sont « débrouillés » en ayant recours à ce que l'on nomme des "stratégies de communication." La définition de la notion de "stratégie de communication » utilisée ici est empruntée à E. Tarone (1983) :

communication strategies are used to compensate for some lack in the linguistic system, and focus on exploring alternate ways of using what one does know for the transmission of a message, without necessarily considering situational appropriateness.

Le manque d'adéquation avec la situation et d'adhésion aux règles d'un genre provient majoritairement de stratégies d'évitement manifestées par des formulations elliptiques ou tronquées. Cette inadéquation a été une cause majeure de rejet des articles. En effet, aucune erreur majeure de lexique ou de syntaxe n'a été relevée. L'inadéquation relevait donc du domaine discursif et non du linguistique au sens étroit du terme. Au-delà des aspects mécaniques de l'usage de la langue, on touchait au cheminement d'une démonstration abstraite et à l'articulation de concepts selon les exigences de textualisation spécifiques d'une langue étrangère.

Malgré les nombreuses différences entre les deux groupes, les deux articles ont finalement été refusés pour des raisons similaires. Les évaluateurs ont décrit ces raisons dans les termes suivants : «discontinuité dans le processus argumentatif », «manque de cohérence stylistique" et "incapacité à introduire, relier et conclure de manière convaincante différents éléments dans plusieurs parties de la démonstration ». En fait, les deux attitudes vis-à-vis de la langue, bien que différentes, ont abouti au même résultat.

Le groupe d'anciens ne s'est jamais vraiment intéressé aux aspects formels de la langue car ces rédacteurs étaient fortement convaincus que le contenu thématique qu'ils avaient à communiquer avait une telle force qu'il l'emporterait sur l'aspect rhématique. Comme le fait remarquer l'un des chercheurs plus âgés : " les faits et les chiffres parlent d'euxmêmes ». Ce désintérêt pour la syntaxe corrobore la remarque de S. Ervin-Tripp, cité par J. C. Richard et G. P. Sampson (1974 : 10), selon laquelle :

the adult's strategies of language learning may be more vocabulary oriented than syntactic. Acquisition of syntax poses a task for the adult which is not easy.

Le groupe de jeunes chercheurs a, quant à lui, démontré les limitations d'une démarche communicationnelle qui ne prend pas assez en compte l'analyse du registre et la cohérence textuelle.

L'orientation «communication d'abord, forme ensuite» s'applique mieux à l'oral (existence d'éléments non-verbaux nombreux, immédiateté de l'information en retour, etc.) surtout lorsqu'il s'agit d'un genre écrit qui n'est enseigné que très rarement en EST au stade de la formation initiale.

\section{Exemples de productions écrites}

Dans leur introduction, le groupe d'anciens a adopté une démarche assez classique en trois étapes :

14 1) identifier le besoin d'une méthode efficace pour régler un problème bien connu et répandu.

2) définir son propre travail en référence aux travaux antérieurs menés par d'autres spécialistes du domaine. 

méthode.

Dans l'exemple qui suit, l'étape $\mathrm{n}^{\circ} 3$ consistait en une description plutôt neutre des méthodes antérieures dans laquelle les avantages contrebalançaient les désavantages. La conséquence était que les méthodes antérieures étaient présentées sous un jour finalement pas défavorable :

\section{Exemple $\mathbf{n}^{\circ} 1$ : incohérence argumentative}

The 'locked boundary modes' method: This method initiated by H...and G... uses sub-structural modes with locked boundary conditions, i.e. the modes of each of them are calculated with clamped boundary conditions. This method has been widely developed and extended to mechanical systems. The latest innovation by L... has made it possible to display the neglected residual term in the frequency analysis...

Hélas, les intentions des rédacteurs étaient précisément de démontrer les limites de la méthode antérieure et de mettre en avant leur propre méthode. Selon les deux chercheurs questionnés par le professeur d'EST, cette attitude ambiguë a été mise sur le compte :

d'une maîtrise insuffisante de la langue qui ne nous aurait pas permis d'exprimer des jugements négatifs, sans parler de critiques caractérisées, formulés avec les nuances nécessaires pour ne pas paraître grossiers [sic] et risquer de s'aliéner définitivement des collègues chercheurs étrangers.

Ce phénomène a entraîné les évaluateurs à reprocher aux rédacteurs un «manque de cohérence » et à déplorer des « introductions inadéquates ».

Dans le cas précis de cet article, le rejet a été dû davantage à l'argumentation incohérente qu'à des stratégies de compensation comme un recours excessif au langage mathématique. (Cette dernière stratégie est traitée plus loin dans cette étude.)

21 L'enseignement de remédiation qui a été adopté n'a pas porté sur les différentes façons de sous-entendre l'inadéquation chez d'autres mais plutôt sur la façon de présenter des phénomènes ou des situations insatisfaisantes et d'y remédier. L'attention s'est déplacée de la personne vers l'objet que les auteurs ont eu plus de facilité à décrire en termes défavorables. La démarche pédagogique a donc dépassé une gestion stricte des éléments linguistiques.

Exemple $n^{\circ} 2$ : incohérence par confusion de registres et contamination du style écrit par le style oral

Consequently within each load increment $\{\mathrm{R}\}$, the gradient matrices [UsG] and [UsF] are computed at the end of the previous step These matrices entirely define the tangential stiffness matrix. Using previous incremental relations, the increase of the displacements, strains (total, elastic and plastic) and stresses, can then be calculated. This we have finally achieved by a simple algorithmic solution: a Euler scheme in which the exact curve is approximated by a series of straight lines, with a slope equal to the start of the increment. Another variant we have thought of uses constant stiffness for each load increment but provides a correction procedure for the out-ofbalance modal forces at the next increment or iteration. être fait preuve ici d'une confiance en soi exagérée. Ils ont enfreint une règle auto- 
imposée d'impersonnalité : «we have finally achieved». De plus,ils ont eu recours à du style oral retranscrit (ils ont aussi, incidemment, démenti la remarque de O. Régent [1985 : 105-120] selon laquelle les chercheurs français n'étaient pas assez orientés vers un objectif-target-oriented).

Cependant, bien que le style ne soit pas conforme au genre de l'article de recherche, les auteurs ont, dans ce cas précis, pris des risques : ils ont structuré leur raisonnement grâce à des marqueurs de cohésion (consequently, this, utilisés comme pronoms en début de phrase) qui contribuent à recréer par écrit le déroulement dynamique du processus scientifique expérimental.

\section{Motivation instrumentale et motivation intégrative}

Les deux définitions de R. Gardner et W. Lambert, ont été utilisées pour définir les deux groupes de chercheurs. Ces définitions somme toute assez statiques trouvent une dimension dynamique chez S. P. Corder qui analyse les deux stratégies de communication offertes à l'apprenant ou à l'utilisateur :

The learner will sometimes wish to convey messages which his linguistic resources do not permit him to express successfully. When in the course of interaction the learner finds himself faced with this situation, he has only two options open to him. He can either tailor his message to the resources he has available, that is, adjust his ends to his means. These procedures we can call message adjustment strategies,or risk avoidance strategies. Or he can attempt to increase his resources by one means or another in order to realize his communicative intentions. These strategies we can call resource-expansion strategies. These are clearly 'successoriented' though risk risk-running strategies. (1983:17)

À partir des définitions de R. Gardner et W. Lambert d'une part et de S. P. Corder d'autre part, l'étude a essayé de répondre à trois questions :

1) Les utilisateurs motivés "instrumentalement " ont-ils recours à des stratégies d'ajustement du message et, par conséquent, évitent-ils le risque ?

2) Les utilisateurs motivés "intégrativement" adoptent-ils des stratégies d'expansion de ressources et prennent-ils donc plus de risques de commettre des erreurs?

3) La motivation vis-à-vis d'une langue étrangère résulte-t-elle en un type de texte spécialisé différent?

Pour répondre, il a fallu vérifier si les différentes stratégies de communication attribuées par S. P. Corder à l'une ou l'autre catégorie d'utilisateurs avaient été adoptées par nos deux groupes-témoins de chercheurs.

Il faut signaler que ces deux groupes avaient choisi de rédiger leurs articles directement en anglais, selon les conseils des enseignants linguistes de l'école.

31 Ils étaient partis d'un canevas assez souple qui consistait en une série de calculs et la description d'un ou deux modèles physiques. Selon les propres termes de l'équipe plus âgée, « la ligne de raisonnement mathématique » avait été exploitée au maximum de sa capacité d'expression.

Il faut remarquer que dans la presque totalité des domaines d'application scientifiques, les chercheurs français ont la réputation d'avoir recours aux équations dans de plus grandes proportions que leurs collègues d'autres nationalités. 

supports non verbaux (si on peut dire que les équations sont non-verbales) avaient été conçus pour être les plus explicites et, ils l'espéraient, aussi autosuffisants que possible. Il s'agit ici d'une attitude de stratégie globale de communication.

Le professeur d'EST n'avait donc pas de version française à partir de laquelle travailler. La méthodologie employée s'inspira donc de celle mise en oeuvre par T. Vàradi (1980). Le professeur et les apprenants partageant la même langue maternelle, ces derniers fournissent un « message optimal » reposant lui-même sur une « signification optimale ».

Les deux groupes ont donc retracé oralement, et en français, tous les stades et articulations de leur argumentation en détail avec le professeur d'EST. Les différentes stratégies ont donc toutes été identifiées et évaluées par rapport au « message optimal ». Selon les catégories de S. P. Corder, les résultats suivants ont été observés.

\section{Tableau 1. Résultats}

\begin{tabular}{|l|l|l|}
\hline Stratégies d'ajustement au message & $\begin{array}{l}\text { Groupe 1 (anciens) en } \\
\text { pourcentage arrondi } \\
\text { Analyse de 270 phrases }\end{array}$ & $\begin{array}{l}\text { Groupe 2 (jeunes) en } \\
\text { pourcentage arrondi } \\
\text { Analyse de 270 phrases }\end{array}$ \\
\hline Évitement du sujet & $6 \%$ & $2 \%$ \\
\hline $\begin{array}{l}\text { Évitement sémantique (exprimer quelque } \\
\text { chose de légèrement différent) }\end{array}$ & $10 \%$ & $6 \%$ \\
\hline $\begin{array}{l}\text { Réduction du message (exprimer moins } \\
\text { ou de façon moins précise) }\end{array}$ & $37 \%$ & $6 \%$ \\
\hline Abandon du message & Aucun & Aucun \\
\hline Stratégies d'expansion des ressources & fungage \\
\hline $\begin{array}{l}\text { Emprunts (quelques mots de français } \\
\text { avec ou sans anglicisation) }\end{array}$ & $3 \%$ & $3 \%$ \\
\hline $\begin{array}{l}\text { Alternance de code } \\
\text { a) introduction d'une phrase complète en } \\
\text { français }\end{array}$ & Aucun & Aucun \\
\hline $\begin{array}{l}\text { Alternance de code } \\
\text { b) introduction } \\
\text { mathématique au milieu de phrases ou } \\
\text { de paragraphes }\end{array}$ & $6 \%$ co & $10 \%$ \\
\hline Paraphrase ou circonlocution & $\begin{array}{l}\text { Supports paralinguistiques } \\
\text { a) langage mathématique }\end{array}$ & $6 \%$ \\
\hline
\end{tabular}




\begin{tabular}{|l|l|l|}
\hline Supports paralinguistiques & $4 \%$ & $6 \%$ \\
b) tableaux, graphes et autres & & \\
illustrations & & \\
\hline
\end{tabular}

36 La stratégie d'abandon de message s'appliquait, pour S. P. Corder, surtout à la communication orale. Il était évidemment impossible pour nos rédacteurs de laisser une phrase imprimée inachevée. Toutefois, une autre forme d'abandon de message peut être détectée dans des phrases commençant en langue générale et se terminant en langage mathématique. Ces occurrences sont enregistrées sous « alternance de code, b) »

37 Les chiffres des supports paralinguistiques b) reposent sur la quantité de langue générale et de langue mathématique remplacée.

38 Si l'on en juge par les chiffres, la démarche du Groupe 1 (anciens) relève plus typiquement de l'évitement du risque.

Tableau 2. Micro-stratégies du Groupe 1

\begin{tabular}{|c|c|c|}
\hline Signification optimale & Version de l'article & Traduction optimale \\
\hline \multirow[t]{2}{*}{$\begin{array}{l}\text { Cette méthode utilise les modes } \\
\text { de chaque sous-structure } \\
\text { calculés en considérant les } \\
\text { frontière de chacune comme } \\
\text { encastrées }\end{array}$} & Évitement du sujet & $\begin{array}{l}\text { This method uses each sub- } \\
\text { structure mode calculated as if } \\
\text { individual borders were } \\
\text { embedded }\end{array}$ \\
\hline & Évitement sémantique & \\
\hline \multirow[t]{2}{*}{$\begin{array}{l}\text { Trois catégories de modes } \\
\text { peuvent être envisagés; nous } \\
\text { allons développer de façon plus } \\
\text { précise la méthode des modes } \\
\text { libres en montrant les } \\
\text { améliorations qu nous } \\
\text { apportons. }\end{array}$} & $\begin{array}{l}\text { Three types of modes are } \\
\text { currently used but the "free } \\
\text { modes" method seems more } \\
\text { precise and can even be } \\
\text { improved. This is what we } \\
\text { intend to demonstrate. }\end{array}$ & $\begin{array}{l}\text { Three classes of modes can be } \\
\text { considered; we intend to } \\
\text { develop more precisely the } \\
\text { free mode method and } \\
\text { demonstrate the } \\
\text { improvements it can offer; }\end{array}$ \\
\hline & Réduction du message & \\
\hline $\begin{array}{l}\text { On peut se poser des questions } \\
\text { sur la complétude de la famille } \\
\text { de déformées utilisée car il y a } \\
\text { surabondance des modes basse } \\
\text { fréquence au détriment des } \\
\text { modes de fréquences associées } \\
\text { plus élevées. }\end{array}$ & $\begin{array}{l}\text { The completeness of the } \\
\text { modes used is questionable. }\end{array}$ & $\begin{array}{l}\text { The completeness of the class } \\
\text { of derived modes is } \\
\text { questionable because there is } \\
\text { an excess of low frequency } \\
\text { modes at the expense of the } \\
\text { associated higher frequency } \\
\text { modes. }\end{array}$ \\
\hline
\end{tabular}

39 Le tableau 2 donne un exemple de chacune des trois micro-stratégies utilisées par ce groupe. La version de signification optimale n'a jamais été rédigée mais a été proposée oralement au professeur d'EST pendant la phase d'analyse rétroactive. La version de l'article correspond à un passage considéré comme inadéquat dans l'article écrit. 
40 Cette inadéquation était considérée par l'évaluateur comme une étape manquante dans une démonstration (évitement du sujet), ou comme une construction ambiguë du fait de l'évitement sémantique (Qu'avons-nous l'intention de démontrer? Que trois types de modes sont actuellement utilisés? Que la méthode des modes libres semble être la plus précise?) ou, encore, du fait d'une information incomplète découlant d'une réduction excessive du message.

41 Le groupe 1 (anciens) se conforme assez au modèle d'évitement du risque, à l'ajustement du message et aux utilisateurs aux motivations « instrumentales » à cause de sa tendance marquée à réduire son message.

42 Le groupe 2 (jeunes) ne se caractérise cependant pas entièrement par la prise de risque et l'expansion des ressources. On a vu que des stratégies d'évitement du sujet, d'évitement sémantique et de réduction du message existaient aussi chez lui, bien que dans de moindres proportions. Le tableau I montre que les emprunts au français sont aussi fréquents dans les deux groupes, et si le passage à une autre langue (alternance de code) au sein d'un certain type de discours est une preuve d'un désir d'expansion de ses ressources, le Groupe 1 est statistiquement plus proche de cette attitude avec son emploi plus fréquent d'un discours hybride langue générale/langage mathématique.

43 Nous avons aussi vu que le Groupe 2 était définitivement plus enclin à utiliser la circonlocution ou la reformulation que le Groupe 1. Cette caractéristique appartient selon nous aux bons utilisateurs de la langue et augure de progrès pédagogiques ultérieurs.

Le tableau 3 montre plusieurs exemples de micro-stratégies attribuées aux « ajusteurs de message » et pourtant utilisées par le groupe 2 dans leur article.

Tableau 3. Exemples de micro-stratégies

\begin{tabular}{|l|l|l|}
\hline Signification optimale & Version de l'article & Traduction optimale \\
\hline \hline sable renforcé & Emprunt (avec anglicisaton) & \\
\hline périmètre & renforced sand & reinforced sand \\
\hline contiguë & perimetre & perimeter \\
\hline $\begin{array}{l}\text { Dans la majorité des cas les } \\
\text { temps de calcul que } \\
\text { nécessite le traitement d'un } \\
\text { ouvrage sont prohibitifs. }\end{array}$ & In most cases CPU* time is prohibitive. & $\begin{array}{l}\text { In most cases, the } \\
\text { calculating time needed } \\
\text { for a constructive } \\
\text { program } \\
\text { prohibitive. }\end{array}$ \\
\hline $\begin{array}{l}\text { contiguous } \\
\text { and }\end{array}$ \\
\hline
\end{tabular}




\begin{tabular}{|l|l|l|}
\hline $\begin{array}{l}\text { L'objet de ce travail est de } \\
\text { proposer une formulation } \\
\text { plus précise des éléments } \\
\text { de base. }\end{array}$ & $\begin{array}{l}\text { The aim of this study is to present a } \\
\text { more accurate formulation of the basic } \\
\text { elements, one that will be more precise } \\
\text { and reduce computational effort. }\end{array}$ & $\begin{array}{l}\text { The aim of this study is } \\
\text { to suggest a more } \\
\text { accurate formulation of } \\
\text { the basic elements. }\end{array}$ \\
\hline & *CPU : Computer Programming Unit & \\
\hline
\end{tabular}

Les emprunts interviennent en nombre limité et sont dictés, dans presque tous les cas, par des ressemblances étymologiques entre le français et l'anglais ou par l'existence de calques ou semi-calques (périmètre/perimeter).

Questionnés à ce sujet les membres du groupe 2 ont invoqué l'inattention, un comportement non partagé par les évaluateurs qui ont réagi systématiquement de manière négative à ce genre d'erreurs.

L'alternance (switching) n'a jamais été en direction d'un mot français ou d'une phrase mais plutôt en faveur d'une abréviation, comme pour «CPU» ou pour un mode non verbal comme une illustration. La réduction du message, souvent par une simplification (voir tableau I), est cependant la stratégie préférée des deux groupes. Le processus de simplification est plus expéditif lorsque l'on omet ou ne mentionne pas.

Selon M.P. Jain, les apprenants, après plus de 20 ans d'apprentissage de la langue,

have reached a stage when they are no longer 'testing their hypotheses' about the second language; they have arrived at a system whatever it is.... There are areas of undeterminacy in (the learner's) syllabus which give rise to systematicunsystematic errors. (1974:203)

Comme nos rédacteurs savaient que, d'une part, des pans entiers de leurs systèmes linguistiques étaient incontrôlables ou absents et que, d'autre part, ils ne pouvaient plus accorder ni le temps ni l'attention nécessaires pour combler ces lacunes, ils ont appliqué la vieille règle : « dans le doute, abstiens-toi ».

\section{L'utilisation du langage mathématique : une micro- stratégie}

One might indeed argue that non-verbal modes of communicating (formulae, diagrams, charts, graphs, and so on) to some degree, at least, represent the basic elements or the 'deep structure' of scientific discourse of which different linguistic textualizations are the surface variants. (Widdowson 1979 : 54)

L'un des aspects principaux de l'analyse rétroactive a été la quantification de la langue générale (LG) par rapport à la langue mathématique (LM). Cette démarche découlait d'une question du groupe 1 (anciens) qui, évidemment plus à l'aise en LM qu'en LG, souhaitait connaître les limites d'un usage intensif de la LM par rapport à ce que pratiquait le reste de la profession, en particulier leurs collègues anglophones.

Une comparaison, entre l'usage de la LM par des chercheurs francophones et par leurs collègues anglophones dans la même spécialité pouvait donc peut-être informer sur le degré de confiance et de maîtrise de la LG de la part des non-natifs. 


\begin{tabular}{|l|l|l|l|}
\hline Calcul des structures & & Géotechnique & \\
\hline \hline Groupe 1 & 4,8 & Groupe 2 & 17,4 \\
\hline 21 articles par natifs ou quasi-natifs & 10,1 & 16 articles par natifs ou quasi-natifs & 15,3 \\
\hline
\end{tabular}

Une comparaison des taux des signes de LM/LG entre les deux groupes n'aurait aucun sens puisque deux domaines de spécialité scientifique peuvent recourir à des proportions différentes de représentation mathématique.

53 Toutefois, il faut noter que dans leurs nombreux articles publiés en français, les rédacteurs du Groupe 1 recouraient moins à la LM que lorsqu'ils rédigeaient en anglais (environ $20 \%$ de moins). Il s'agissait donc bien là d'une stratégie de rédaction.

Bien que les deux articles aient été rejetés par des évaluateurs agissant pour le compte de deux revues différentes, on peut noter que, au moins, le Groupe 2 se conformait mieux que le Groupe 1 à une exigence quantitative du genre rédactionnel dans leur spécialisation.

Une différence fondamentale d'attitude entre les deux groupes réside donc dans le recours plus systématique du Groupe 1 à la LM. Du propre aveu du Groupe 1, la LG n'était souvent employée que lorsque la LM ne pouvait rendre compte à elle seule de l'ensemble de la démonstration.

Un exemple typique de recours excessif à la LM est ce passage considéré comme obscur par un évaluateur (et sans doute plus encore par le professeur d'EST, bien que pour des raisons différentes) :

...The equations of motion for the whole structure using Lagrange equations can be written as follows for each substructure :

$[\mathrm{M} \mathrm{j}]\{\mathrm{xj}(\mathrm{t})\}+[\mathrm{Kj}]\{\mathrm{xj}(\mathrm{t})\}-[\mathrm{R} \mathrm{j}] \mathrm{T}\{\mathrm{l}(\mathrm{t})=\{\mathrm{fj}(\mathrm{t})\}$

for $\mathrm{j}=1,2, \ldots . \mathrm{N}$

$\left.{ }^{*}\right)$ Damping is supposed negligible and matrices [Kj] and [Mj] are not assumed to be symmetrical. The extension of the method to symmetrical or non-symmetrical couplings between velocities presents no difficulties.

(**) We can also assume that the exciting forces are harmonic and we work in a harmonic steady state so that $\{\mathrm{xj}\},\{f \mathrm{j}\},\{\mathrm{lj}\}$ designate algebraic magnitudes.

Lorsque la signification optimale de la phrase $\left(^{*}\right)$ a été demandée en français au Groupe 1, la réponse a été :

Afin de ne pas alourdir les expressions des équations matricielles qui sont développées dans la suite, l'amortissement est supposé négligeable...

La réduction de message tenait à l'ignorance d'une seule notion lexicale : «alourdir ». La phrase «in order to" = "afin de", était si bien connue des rédacteurs qu'ils la considéraient comme appartenant à la LM.

Pour la phrase (**), la signification optimale a été : 
Afin encore de simplifier les calculs qui suivent, nous supposerons que les forces etc.

From the previous equations (8) we obtain new equations representative of the motion of the structure in the low frequency range. In the first stage, each modal matrix is split into kept and lost modes associated respectively to parameters $\{q \mathrm{kj}\}$ and $\{\mathrm{qlj}\}$, i.e. masters and slaves.

For substructure $\mathrm{j}$ we then have:

(equations)

and for the whole structure:

(equations)

Lorsque le collègue d'EST leur a demandé de développer et de compléter le passage, le Groupe 1 proposa la version optimale suivante qui fut traduite par :

We are seeking approximate equations obtained from the previous ones which would be representative of the structure behaviour in low frequencies. Each modal matrix on the right and left is partitioned into kept modes which will make up the truncated modal basis, and lost modes associated respectively to the generalised parameters $\{\mathrm{qkj}\}$ and $\{\mathrm{q} \mathrm{lj}\}$, in which $\mathrm{k}$ relates to the kept modes and $\mathrm{l}$ to the lost modes. We shall call them respectively masters and slaves. For substructure «j», we then have: (equations)

Pas vraiment la même histoire...

Le tableau 4 donne les formules d'introduction, de transition et de conclusion choisies pour accompagner les équations. Seules les formes à taux d'occurrence supérieur à $1 \%$ ont été retenues.

Tableau 4. Formules d'introduction, de transition et de conclusion choisies pour accompagner les équations

\begin{tabular}{|l|l|l|}
\hline Introductory & intermediary & Concluding \\
\hline $\begin{array}{l}\text { (Equation) is written } \\
\text { as... }\end{array}$ & .. when... & $\begin{array}{l}\text {..this is but (a possible/ rough/etc. } \\
\text { explanation) }\end{array}$ \\
\hline $\begin{array}{l}\text { We can write... } \\
\text { Let us consider... } \\
\text { (Equation) can also be } \\
\text { written as follows: }\end{array}$ & \begin{tabular}{l}
...we cobtain... (is)(also) written as... \\
\hline
\end{tabular} & \\
\hline
\end{tabular}




\begin{tabular}{|l|l|l|}
\hline $\begin{array}{l}\text { We designate... } \\
\text { (Equation) lead(s) to } \\
\text { the following: }\end{array}$ & $\begin{array}{l}\text {..(eventually/then) } \\
\text { becomes.. }\end{array}$ & $\begin{array}{l}\text { The complete solution/ } \\
\text { demonstration is to be } \\
\text { found in (name of researcher) }\end{array}$ \\
\hline $\begin{array}{l}\text { We then have what } \\
\text { follows... } \\
\varnothing \text { (no introd. form) }\end{array}$ & $\begin{array}{l}\text {......... can the other hand... } \\
\text { as... }\end{array}$ & \\
\hline \hline $\begin{array}{l}\text { Which may be better (! expressed } \\
\text { (...with.... }\end{array}$ & \\
Expressed as... . & ..we assume... & \\
\hline
\end{tabular}

66 Le tableau 5 résume les principales fonctions que peut ou non assurer la langue mathématique.

Tableau 5. Langue mathématique

\begin{tabular}{|c|c|}
\hline PRINCIPALES FONCTIONS ASSURÉES : & $\begin{array}{lllll}\text { PRINCIPALES } & \text { FONCTIONS } & \text { NON } & \text { OU } & \text { MAL } \\
\text { ASSURÉES } & & & & \end{array}$ \\
\hline $\begin{array}{l}\text { transmission des notions de : } \\
\text { - taille } \\
\text { - dépendance/indépendance } \\
\text { - imbrication } \\
\text { - progression/régression } \\
\text { - augmentation/diminution (régulière ou } \\
\text { non, prédictible ou aléatoire) } \\
\text { - itération } \\
\text { - interruption } \\
\text { et......conclusion inattaquable }\end{array}$ & $\begin{array}{l}\text { - introductions et transitions } \\
\text { - coordination } \\
\text { - subordination } \\
\text { - causalité } \\
\text { - hypothèse restrictive } \\
\text { - présentation du déroulement chronologique } \\
\text { d'un processus } \\
\text { - définition de l'environnement physique d'une } \\
\text { expérience ou d'un procédé }\end{array}$ \\
\hline
\end{tabular}

Dans le cas du Groupe 1, la croyance en l'omnipotence et l'autosuffisance de la LM a été infirmée. L'observation des stratégies de communication des deux groupes a révélé "négativement", ou par défaut, les formes linguistiques non assimilées. Elle a aussi souligné le lien étroit existant entre analyse du discours et analyse de l'erreur.

Ces fonctions utiles assumées par la LM ont permis aux rédacteurs d'éviter quelques pièges traditionnels de la grammaire anglaise, comme les comparatifs/superlatifs (is bigger/smaller than), les prépositions et particules (is a function of, is included in, is equal to, is not present in, etc.)

Un certain nombre d'erreurs lexicales ont aussi été commises lorsque des substituts aux symboles mathématiques ont dû être inclus en $L G$ (par ex. $\Rightarrow>$ remplacé par la forme «leads at »). 


\section{Conclusion : portée et limitations de l'étude}

70 Les rédacteurs du Groupe 1 se sont caractérisés surtout par le souci d'éviter le risque. En tant qu'utilisateurs "motivés instrumentalement", ils se sont conformés à la démarche générale d'ajustement du message définie par S. P. Corder surtout par leur attitude de réduction du message.

71 Les rédacteurs du Groupe 2 se sont révélés plus enclins à augmenter leurs ressources et à prendre des risques, surtout lorsqu'ils ont choisi de paraphraser, reformuler ou utiliser des circonlocutions en LG.

Les deux groupes sont apparus comme désireux surtout de simplifier leur message et ont choisi de le faire en utilisant spontanément des stratégies d'évitement. L'« autopsie » des articles a aussi révélé que la communication non équivoque était souvent obtenue au prix de l'abandon de la signification optimale.

73 L'absence, pour ces deux générations de chercheurs, de formation spécifique et systématique EST/rédaction d'articles de recherche a été ressentie comme un manque. Cette absence de formation linguistique spécifique a conduit les utilisateurs à recourir à des stratégies rédactionnelles personnelles qui ne relèvent pas toutes de catégories linguistiques ou pédagogiques traditionnellement identifiées et enseignées par des professeurs linguistes (par exemple, l'omission qui s'apparenterait au silence dans la communication orale).

74 La formation EST en remédiation post hoc et dans une situation psychologique d'échec (refus de publication) a été reçue et utilisée différemment par les deux groupes selon, encore, leur "héritage pédagogique». Le Groupe 1 continue à fréquenter l'institut médico-légal du professeur d'EST, tandis que le Groupe 2 est désormais autonome pour la rédaction de ses articles.

Bien que réduit, l'échantillonnage des sujets humains et thématiques de l'étude a permis néanmoins d'identifier des comportements rédactionnels types. D'autre part, l'expérience d'autres actions de remédiation linguistique du type de celui de cette étude a fait apparaître que la population de chercheurs de l'ECN et de ses laboratoires du CNRS associés (plus de 200 personnes au total) se répartissait à peu près selon ces deux types de rédacteurs, autant pour ce qui est de l'âge que des stratégies et des styles rédactionnels en langue anglaise.

Pour être pleinement utile et pertinente, l'intervention du spécialiste de langue devrait pouvoir dépasser le strict domaine de la langue et de l'analyse des items linguistiques traditionnels. Le professeur linguiste devrait être compétent pour ce qui est de l'intermodalité de systèmes sémiotiques différents: pouvoir déterminer comment interagissent la langue naturelle, les langues formelles, les schémas et les représentations graphiques lorsque ces différents systèmes sémiotiques sont associés pour rendre visibles et explicites des relations.

Se pose aussi le problème des traductions, non pas d'une langue à l'autre, mais d'un registre sémiotique à un autre : texte, tableau, graphe, etc. Ces aspects constitueront pour nous l'étape suivante dans l'étude des stratégies rédactionnelles des scripteurs non linguistes. 


\section{BIBLIOGRAPHIE}

Corder, S. P. 1983. « Strategies of communication ». In Faerch, C. \& Kasper G., Strategies in Interlanguage Communication. Harlow : Longman.

Ervin-Tripp, S. 1970. « Structure and process in language acquisition ». In Alatis J.E. (dir.), Monograph Series on Languages and Linguistics. Washington, D.C. : Georgetown University Press, 313-344.

Faerch, C. \& G. Kasper. 1983. Strategies in Interlanguage Communication. Applied linguistics and Language Study. Harlow : Longman.

Gardner, R. \& W. Lambert. 1959. « Motivational variables in second language acquisition ». Canadian Journal of Psychology 13, 266-272.

Jain, M.P. « Error analysis: Source, cause and significance ». In J.C. Richards (ed.), 1974.

Régent, $\mathrm{O}$. « A comparative approach to the learning of specialized written discourse ». In P. Riley (ed) Discourse and Learning, London : Longman, 1985.

Richards, J. C. \& G. P. Sampson. 1974. « The study of learner English ». In Richards, J. C. \& G. P. Sampson (dir.), Error Analysis: Perspectives on second language acquisition. Harlow : Longman.

Richards, J. C. (dir.).1974. Error Analysis: Perspectives on second language acquisition. Harlow : Longman.

Tarone, E. 1983. « Some thoughts on the notion of 'communication strategy' ». In Faerch, C. \& G. Kasper, G. (dir.).

Vàradi, T. 1980. «Strategies of target language learner communication: message adjustment ». IRAL 18, 59-71.

Widdowson, H.G.1979. The Description of Scientific Language. Explorations in Applied Linguistics. Oxford: Oxford University Press.

\section{ANNEXES}

Un résumé de l'étude désormais classique de Tamàs Vàradi, Strategies of target language communication: message adjustment est fourni par Faerch \& Kasper (1983 : 74) :

Vàradi establishes a model of interlanguage production which focusses on the strategies the learner employs when he experiences a 'hiatus' in his interlanguage repertoire. In order to adjust his message to his communicative resources, the learner either replaces the meaning or form of his intended message by using items which are part of his interlanguage, or he reduces his intended message on either the formal or the functional level. This model was tested out in a pilot study involving adult Hungarian learners of English at the intermediate level. The experiment confirmed the hypothetical model of adjustment strategies. Although the pilot study reported on deals with learners' written performance exclusively, Vàradi emphasizes that in order to assess the communicative 
effect of learners' utterances more precisely, they must be placed into an interactional perspective involving the native speaker's reception and responses.

\section{RÉSUMÉS}

L'article rend compte d'une analyse de corpus limité mais représentatif de stratégies et de styles mis en œuvre par deux générations de scientifiques français (pré et post enseignement communicationnel d'une langue) lors de la rédaction d'articles de recherche devant être publiés par des journaux spécialisés anglophones. L'accent est mis sur les stratégies de communication relevant plus particulièrement de deux attitudes différentes en apprentissage des langues étrangères : utiliser la langue en tant qu'outil servant à satisfaire précisément les exigences d'une seule tâche spécifique (une sorte d'attitude "d'utilisation puis d'oubli»), et tirer un profit maximum de toutes les situations langagières rencontrées pour développer ses ressources et améliorer sa capacité globale à utiliser la langue. L'article étudie aussi certains aspects du traitement des relations qui existent entre la langue générale de l'argumentation et le «noyau dur » du langage mathématique représenté par les équations et les formules.

The paper presents the analysis of a corpus which, although limited, is hopefully representative of the styles and strategies used by representatives of two generations of French scientists (preand post-Communicative Language Teaching) in the writing of research articles to be published in specialised Anglophone journals. The focus is on those communication strategies that may be attributable to two fundamentally different attitudes towards foreign language learning: Using the language precisely as a tool to meet the requirements of a specific task only (a sort of "useand-forget" attitude), and taking advantage of all possible situations to try and expand one's resources with a view to improving one's overall performance in the use of the language. The paper also looks at some aspects of the treatment by the two groups of the relationships between general argumentative language and hard-core mathematical language in the form of equations and formulae.

\section{INDEX}

Mots-clés : anglais de spécialité, article de recherche, didactique, évitement, langue générale, mathématiques, natif, non-natif, stratégie rédactionnelle, style rédactionnel

Keywords : avoidance, ELT, English for Specific Purposes, general language, mathematics, native English speaker, non-native English speaker, research article, writing strategy, writing style

\section{AUTEUR}

\section{CLAUDE SIONIS}

